\title{
Marketing del chocolate en pymes de México
}

\author{
MANUELA CAMACHO-GÓMEZ*
}

pp. 93-110

\begin{abstract}
RESUMEN Este artículo se refiere a los componentes básicos identificados en el marketing que utilizan las empresas del cacao y el chocolate en México. La investigación se guió por el enfoque cualitativo fenomenológico. Las pymes identificadas y estudiadas son de origen y capital mexicano, sumaron un total de 21 organizaciones ubicadas en al menos once entidades del país donde destacan las zonas del sureste, occidente y centro. El análisis se desarrolló desde la perspectiva de Jerome McCarthy, quien introdujo el concepto de las 4 P's del marketing. Para este caso, se observa en los hallazgos la presencia predominante de estrategias de marketing en tres P's: producto, promoción y plaza.
\end{abstract}

\section{PALABRAS CLAVE marketing, pymes, chocolate, cultura.}

\author{
COMO CITAR ESTE ARTÍCULO \\ How to cite this article: \\ Camacho-Gómez, M. (2019). \\ Marketing del chocolate en \\ pymes de México. Revista \\ Perspectiva Empresarial, 6(1), \\ 93-110 \\ Recibido: 06 de noviembre de \\ 2018 \\ Aprobado: 11 de febrero de 2019
}

* Doctora en Educación Internacional. Profesora-investigadora de tiempo completo. Universidad Juárez Autónoma de Tabasco, Tabasco, México. E-mail: manuela.camacho@ujat.mx. ORCID: 0000-0003-3632-064X. Google Scholar: https:// scholar.google.com.mx/citations?user=BCnnuEIAAAAJ\&hl=es. 


\section{Chocolate marketing in SMEs of Mexico}

ABSTRACT This paper deals with the basic components identified in the marketing activities carried out by cocoa and chocolate companies in Mexico. The research followed the qualitative phenomenological approach. The SMEs identified and studied are of Mexican capital and origin, totaling 21 organizations located in at least eleven regions of the country, mainly the southeastern, western and central areas. The analysis was developed from the Jerome McCarthy's perspective, who introduced the concept of the 4 P's in marketing. For this case, the prevailing presence of marketing strategies in three P's: Product, promotion and place, is observed in the findings.

KEY WORDS Marketing, SMEs, chocolate, culture.

\section{Marketing do Chocolate em pymes do México}

RESUMO Este artigo se refere aos componentes básicos identificados no marketing que utilizam as empresas do cacau e o chocolate no México. A investigação se guiou pelo enfoque qualitativo fenomenológico. As pymes identificadas e estudadas são de origem e capital mexicano, somaram um total de 21 organizações localizadas pelo menos onze entidades do país onde destacam as zonas do sudeste, ocidente e centro. A análise se desenvolveu desde a perspectiva de Jerome McCarthy, quem introduziu o conceito das 4 P's do marketing. Para este caso, se observa nas descobertas a presença predominante de estratégias de marketing nas três P's: produto, promoção e praça.

PALAVRAS-CHAVE marketing, pymes, chocolate, cultura. 


\section{Introducción}

A nivel mundial las pequeñas y medianas empresas - pymes- son conocidas como unidades económicas que favorecen la generación de empleos. Su clasificación se fundamenta en dos componentes: número de empleados y facturación anual. En México las pymes suman 95000 organizaciones, de las cuales el $83 \%$ son pequeñas y 17 \% medianas (Secretaria de Economía, 2016). En este contexto, el sector agroindustrial posee especial distinción por el tipo de productos que fabrica y comercializa dado que es determinante en el desarrollo de la agricultura mexicana. Al igual que en otros países, en México, las pymes son de gran importancia por su número de unidades y por ser generadoras de empleos. Los criterios para determinar su clasificación difieren de un país a otro, aunque usualmente son dos las bases: la cantidad de personal ocupado y como complemento se considera el volumen de ventas anuales, los ingresos y los activos fijos (INEGI, 2009). Las pymes se refieren a empresas con marcadas diferencias en sus capacidades de organización, comercialización y tecnología, aunque son intensivas en mano de obra (FAO, 2013).

De acuerdo al tamaño de las organizaciones existen singularidades en su desempeño empresarial. Las pequeñas empresas son negocios dedicados al comercio y servicios que contemplan entre 11 y 30 trabajadores o que generan ventas anuales entre 4 y 100 millones de pesos mexicanos MXN-. En tanto que las medianas cuentan desde 31 hasta 100 trabajadores, y producen anualmente ventas que van desde los 100 millones hasta 250 millones de MXN o más (Secretaría de Economía, s.f.). Estas empresas pueden desarrollar su competitividad con base en la mejora continua de su administración y procesos, así como en las habilidades directivas de sus gerentes-propietarios.

Con respecto a su participación económica, según los resultados de la Encuesta Nacional sobre Productividad y Competitividad de las Micro, Pequeñas y Medianas Empresas -ENAPROCErealizada en 2015 por el Instituto Nacional de Geografía -INEGI-, en México existen 4 millones de estas unidades: 3,9 millones de dimensión micro; 79 mil pequeñas y 16 mil medianas. Estas participan con el $63 \%$ del empleo y el $35 \%$ de la producción bruta total (Secretaría de Economía,
2016). En este sentido las pymes agroindustriales tienen una significativa participación del valor añadido total en su sector (FAO, 2013).

Con estas perspectivas, el presente artículo tiene como propósito la identificación y análisis de las características generales del marketing que utilizan las empresas pymes agroindustriales del cacao y el chocolate a través del modelo de las 4 P's (McCarthy, 1960; Perreault and McCarthy, 2002) y con el cual se lograron conclusiones sobre estrategias mercadológicas que podrían abonar al estudio del marketing del chocolate en México.

\section{Pymes agroindustriales}

La agroindustria es la actividad económica que comprende la producción, industrialización y comercialización de productos agropecuarios, forestales y otros recursos naturales donde existe un proceso que comprende: producción agrícola; actividades de tratamiento postcosecha; procesamiento y comercialización nacional e internacional cuyos productos llegan al consumidor final (GobMx, 2017). De acuerdo con la FAO (2013) el sector agroindustrial -también denominado agroprocesamiento- incluye productos para la subsistencia, mercados locales y exportaciones tradicionales diferenciadas que también se orientan a mercados nacionales. En las agroindustrias el valor añadido es visible para los consumidores a través de los empaques de los productos, embalajes, formas de conservación, vida en anaquel, almacenamiento, transporte, disponibilidad y diversificación de los mismos (Camacho, 2014). Se puede decir que este sector tiene un carácter estacional y una variabilidad en la producción, así como propiedades perecederas que exigen actividades a partir de una base agrícola. Por tanto, se debe tomar en cuenta la necesidad de estrategias de comercialización más eficaces (FAO, 1997, como se citó en Camacho, 2018).

Bajo tales premisas, se aprecia que las empresas agroindustriales sean pymes en su mayoría. Las pequeñas empresas por lo general se ubican en áreas rurales; en tanto que las medianas se localizan en las zonas urbanas, aprovechan más las economías de escala y poseen una mejor infraestructura (Quadratín, 2017). Las pymes 
agroindustriales se distinguen por el limitado número de empleados y por el conocimiento profundo de sus gerentes-propietarios sobre el proceso agrícola y el manejo de la producción y operaciones de sus productos, ya que en esas áreas recaen las actividades estratégicas del negocio (Camacho, 2018).

En México las materias primas tradicionales como el café y el cacao son fundamentales en la agroindustria puesto que tienen especial importancia gastronómica, económica, social y cultural. En la industria del cacao se producen alrededor de 28 mil toneladas al año. Los principales Estados productores son Tabasco y Chiapas y el producto derivado más conocido es el chocolate, considerado un elemento de identidad nacional que forma parte de la herencia cultural mexicana (Quadratín, 2017).

\section{Marketing para pymes}

En lo que respecta al marketing esta es un área de las empresas que junto con la producción (operaciones, procesos), las finanzas, administración y recursos humanos generan funcionalidad; no se concibe a una sin las otras, ya que son interdependientes. En esta coyuntura es relevante mencionar que, si bien todas las funciones son indispensables, tres de ellas se operan básicamente hacia el interior; no así el marketing que es la cara empresarial ante diversos públicos (Camacho y
Andrade, 2015). Esta área también se la reconoce como "un proceso por el cual individuos y grupos obtienen lo que necesitan y desean creando e intercambiando productos y valor con otros" (Kotler y Armstrong, 2001, p. 10).

Consecuentemente se requiere tener claro cuáles son las capacidades de marketing en este tipo de empresas. De tal manera que coadyuven a alcanzar posiciones de ventaja ante la competencia al otorgar valor superior a los clientes. El marketing permite que la organización adecúe su esfuerzo comercial hacia el retorno de la inversión previsto para cada tipo de clientes (Talaya et al., 2008, como se citó en Reinares y Blanco, 2015).

A diferencia de las grandes empresas, las pymes privilegian de manera tácita o explícita el modelo del auto-branding; referido a la identidad personal de su administrador y cuyas habilidades identifican al negocio por su interrelación con los clientes, con quienes procura una relación estrecha de largo plazo (Resnick et al., 2016).

Además de las estrategias orientadas al cliente, las pymes también suelen utilizar acciones orientadas al mercado. De esta manera el marketing identifica, evalúa y elige las oportunidades del mercado que puede atender; entonces define las estrategias para aprovecharlas (Kotler y Armstrong, 2008, como se citó en Reinares y Blanco, 2015). De ahí que el valor superior del producto juega un papel contundente para el desarrollo, extensión y diversificación del target de las empresas (tabla 1).

Tabla 1. Estrategias de producto-mercado

\begin{tabular}{|c|c|c|c|c|c|}
\hline & \multirow[t]{2}{*}{$\begin{array}{l}\text { Productos } \\
\text { actuales }\end{array}$} & \multirow[t]{2}{*}{$\begin{array}{l}\text { Mejoras en los } \\
\text { productos existentes }\end{array}$} & \multicolumn{2}{|c|}{$\begin{array}{l}\text { Nuevos productos relacionados } \\
\text { con los actuales }\end{array}$} & \multirow[t]{2}{*}{$\begin{array}{c}\text { Nuevos productos } \\
\text { sin relación con los } \\
\text { actuales }\end{array}$} \\
\hline & & & $\begin{array}{l}\text { Cambios en la } \\
\text { gama }\end{array}$ & $\begin{array}{l}\text { Extensión de la } \\
\text { gama }\end{array}$ & \\
\hline $\begin{array}{l}\text { Mercados } \\
\text { actuales }\end{array}$ & $\begin{array}{l}\text { Estrategia de } \\
\text { penetración }\end{array}$ & $\begin{array}{l}\text { Estrategia de } \\
\text { reformulación }\end{array}$ & $\begin{array}{l}\text { Estrategia de } \\
\text { sustitución }\end{array}$ & $\begin{array}{l}\text { Estrategia de } \\
\text { extensión de } \\
\text { línea de producto }\end{array}$ & $\begin{array}{l}\text { Diversificación } \\
\text { horizontal }\end{array}$ \\
\hline Mercados nuevos & $\begin{array}{l}\text { Estrategia de } \\
\text { desarrollo de } \\
\text { mercado }\end{array}$ & $\begin{array}{l}\text { Estrategia de } \\
\text { extensión }\end{array}$ & $\begin{array}{l}\text { Estrategia de } \\
\text { segmentación }\end{array}$ & $\begin{array}{l}\text { Diversificación } \\
\text { vertical }\end{array}$ & Diversificación lateral \\
\hline
\end{tabular}


Estas estrategias y otras que se consideren viables para el éxito de las pymes deben tener claridad en sus negocios y definir a quiénes está dirigido (Barrow, 1996). Se precisa conocer los atributos diferenciadores de sus productos o servicios y el grupo de compradores reales y potenciales que adquirirán esos bienes en función de las ventajas que ofrecen. Por ello el marketing en las pymes es determinante para dar a conocer, convencer, posicionar, consolidar y recordar a los segmentos la presencia y bondades de un producto tangible o intangible (figura 1).

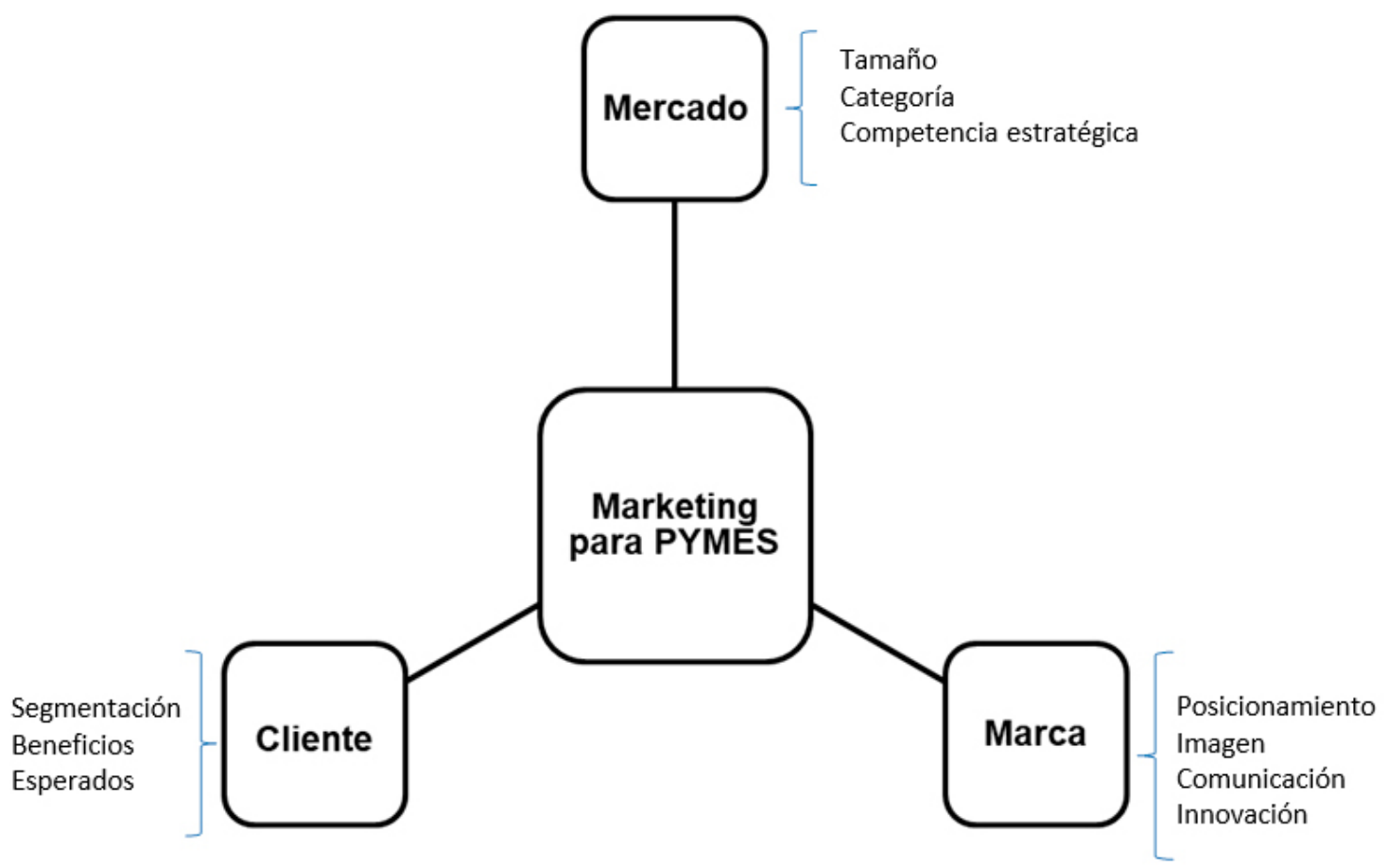

Figura 1. Condiciones de mercados para pymes. Fuente: Marcos (2015).

Se observa que el producto, el precio, la promoción y la plaza (4 P's) tienen igual importancia para una empresa pequeña, mediana o grande. No obstante, en función del tamaño de la organización, cada una de estas P's tiene implicaciones diferentes en su aplicación y alcance.

El producto puede ser la base de la ventaja competitiva de las pymes, pero necesita de una diferenciación efectiva con respecto a los competidores. El precio, por su parte, es influenciado por el público objetivo dado que ellos determinan el potencial valor de un producto o servicio (Equipo Vértice, 2009). Esto implica una política de fijación de precios escrupulosa que permita que la empresa sea competitiva y oriente sus esfuerzos hacia la satisfacción de sus mercados.

Con respecto a la promoción (comunicación) se utilizan estrategias de publicidad, promoción de ventas, merchandising, relaciones públicas y venta personal. El objetivo de las comunicaciones utilizadas por la pymes es informar, persuadir, recordar y crear imagen de marca o de producto para generar actitudes o impresiones tales como prestigio calidad, credibilidad. Esta es la razón por la que primero se debe llevar a cabo una investigación de mercado para delimitar el target y así conocer o confirmar el perfil del consumidor potencial, sus necesidades y aspiraciones. A los responsables del marketing les corresponde 
crear una asociación entre el producto y una imagen atractiva que induzca a la compra del mismo (Equipo Vértice, 2009). La promoción de ventas en las pymes suele ser simple como son las muestras y degustaciones, pero en algunos sectores sus distribuidores (empresas grandes) pueden apoyar mediante una promoción compartida. El merchandising agrupa diversas actividades en los puntos de venta para estimular la compra de determinados productos (Equipo Vértice, 2009; PyME-JICA, 2005).

En lo tocante a la distribución de los productos esto se determina de acuerdo a las características del segmento, las estrategias para definir los canales de distribución idóneos y llegar al punto de venta (Marcos, 2015). La elección de los canales depende de la dimensión del mercado, las tendencias del sector y la forma de la distribución geográfica del público objetivo, los hábitos de compra, las características del producto y el presupuesto disponible por parte de la organización (Equipo Vértice, 2009).

\section{Descripción de la metodología empleada}

El desarrollo de este artículo se deriva de una investigación fundamentada en el enfoque cualitativo fenomenológico, que se caracteriza por indagar las experiencias o fenómenos estudiados (Martínez, 2006). No obstante, se llevó a cabo un análisis intencional sobre el marketing en empresas mexicanas pequeñas y medianas que fabrican y comercializan chocolate. Se eligieron procedimientos y técnicas que permitieron observar repetidas veces los datos estratégicos sobre el marketing implantado por las pymes de referencia. En este contexto, se analizaron páginas Web y redes sociales de las organizaciones estudiadas. Se privilegió, además, la revisión documental, la observación de anuncios publicitarios en medios tradicionales (periódicos, revistas y espectaculares). También se recurrió a la observación directa y observación participativa en las empresas ubicadas en Tabasco. Consecuentemente se apreció la gran variedad y complejidad de las partes (P's del marketing). De tal manera que la réplica de las observaciones en las distintas fuentes tuvieron como propósito protocolar el fenómeno de la realidad estudiada; descrito en su contexto natural, en su situación peculiar y el mundo propio donde se presenta (Martínez, 2006).

Las 21 empresas analizadas fueron identificadas en el proceso de investigación; y para confirmar los datos documentales se replicó la observación directa a través del apoyo de colegas que residen en el norte y el centro del país. El análisis se realizó de acuerdo a las categorías definidas: producto, precio, promoción y plaza; las cuales son utilizadas en el marketing del chocolate mexicano.

\section{Resultados}

En el mercado del chocolate confluyen empresas grandes, medianas, pequeñas y micro; sus principales diferencias estriban en uso de tecnologías, volúmenes de producción, participación en mercados internacionales y el alcance y efectividad de las estrategias de marketing utilizados. Respecto a los consumidores, estos tienden a percibir y en ocasiones a conocer sobre los componentes tangibles e intangibles asociados al producto (tabla 2).

Tabla 2. Componentes tangibles e intangibles del producto chocolate

\begin{tabular}{lll}
\hline & \multicolumn{1}{c}{ Tangibles } & \multicolumn{1}{c}{ Intangibles } \\
\cline { 2 - 3 } Ingredientes & $\begin{array}{l}\text { Marca (conciencia, } \\
\text { aceptación y preferencia }\end{array}$ \\
\cline { 2 - 3 } & Sabor & Prestigio de la empresa \\
\cline { 2 - 3 } & $\begin{array}{l}\text { Calidad real del } \\
\text { producto }\end{array}$ & $\begin{array}{l}\text { Calidad percibida en el } \\
\text { producto }\end{array}$ \\
\cline { 2 - 3 } & $\begin{array}{l}\text { Diseño del } \\
\text { Empaque }\end{array}$ & Publicidad \\
\cline { 2 - 3 } & Color & Innovación \\
\cline { 2 - 3 } & Presentaciones & Investigación y desarrollo \\
\hline Atributos & Precio & Interactividad \\
\hline & $\begin{array}{l}\text { Accesibilidad al } \\
\text { producto }\end{array}$ & Celebridades \\
\hline
\end{tabular}

Fuente: Camacho (2015). 


\section{Pymes mexicanas del chocolate}

Las pymes en México se encuentran presentes en todos los sectores, sin embargo en la agroindustria son muy representativas. En este sector, los grandes corporativos internacionales del chocolate tienen una amplia participación en el mercado nacional; no obstante, las empresas mexicanas también hacen lo propio basando su ventaja competitiva en el arraigo cultural-histórico de la cocina ancestral y en la calidad de sus materias primas e insumos. De acuerdo a la investigación realizada se pudieron localizar 21 pymes mexicanas que se dedican a la fabricación y comercialización de chocolate; su ubicación geográfica predominante es centro, occidente y sureste del país; 19 de ellas se crearon en el período comprendido entre 1860 y 1959, las otras dos organizaciones son más próximas a la época actual.

\section{Marketing del chocolate}

Los hallazgos evidencian que las organizaciones pequeñas y medianas dedicadas a la producción y comercialización del cacao y el chocolate se distinguen por ser cada vez más innovadoras en sus procesos de producción, cuidando los ingredientes e insumos; sin embargo en el marketing aún existen áreas de oportunidad, especialmente porque participan en un mercado muy competido donde incursionan las empresas líderes mundiales del chocolate. Se aprecia que estas empresas desarrollan productos y servicios que crean valor y satisfacen a los clientes de sus mercados y por lo tanto generen ventas y utilidades para la organización (Camacho, 2017).

Igualmente se pudo observar que las características del marketing del chocolate enfatizan sus acciones a través de las 4 P's, teniendo como premisa el mercado objetivo al que dirigen sus esfuerzos. Se distinguen por sus productos de alta calidad, líneas innovadoras y diferenciadas; con marcas individuales o familiares, distribución directa o mediante otros canales. Sus empaques son más bien protectores, aunque las empresas medianas si invierten en su imagen promotora. La garantía es limitada debido a que lo artesanal del producto podría no cumplir con los estándares de inocuidad apropiados a su vida de anaquel (figura 2).

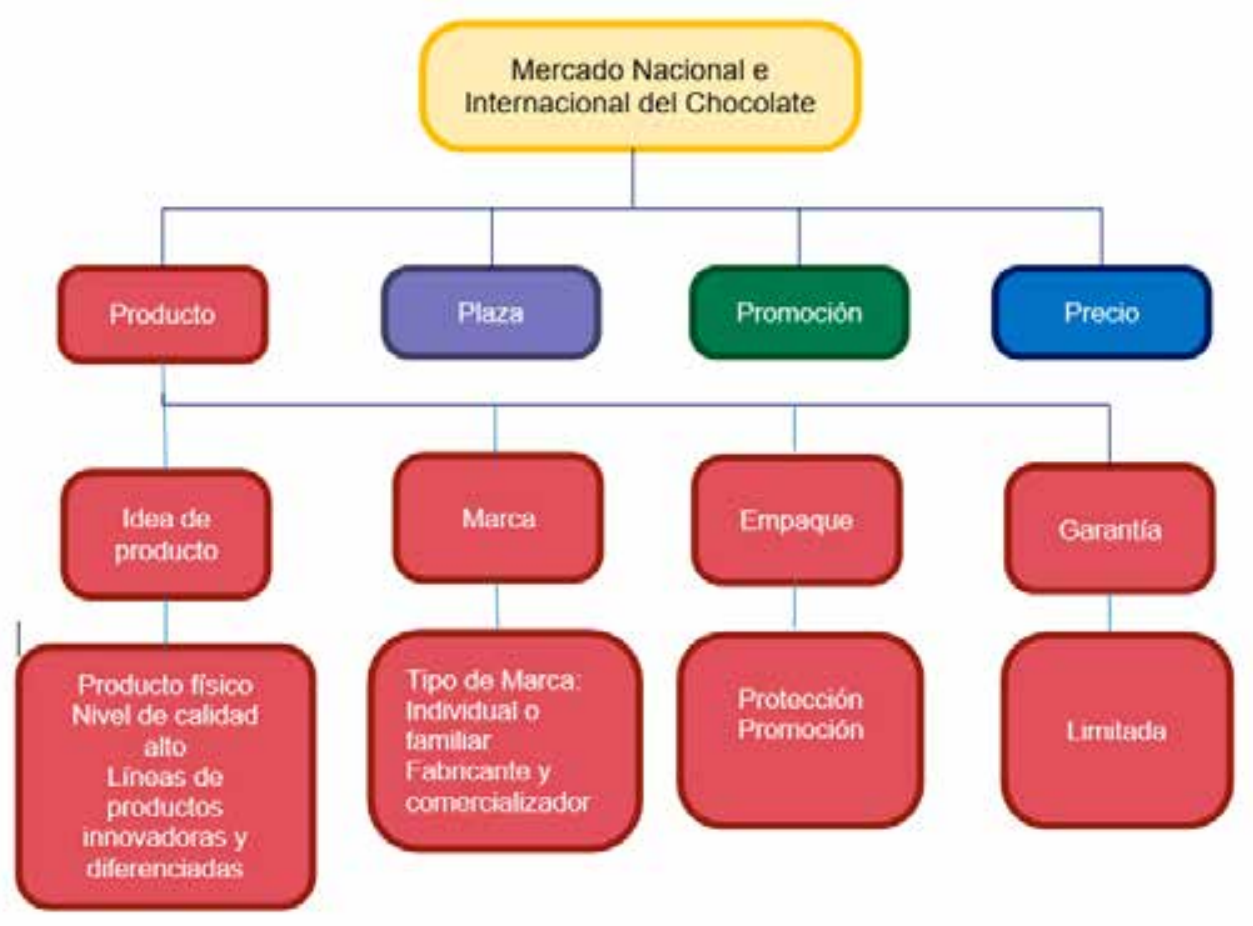

Figura 2. Características de las 4 P's del marketing del chocolate. Fuente: Perreault y McCarthy (2002). 


\section{Producto}

El producto de las pymes es más bien artesanal, predomina el chocolate para mesa en polvo y golosina. La tendencia es la producción y comercialización del chocolate Premium. Las marcas están registradas y el eslogan contiene leyendas asociadas a la tradición, calidad y sabor, además del lugar de elaboración. Respecto a la innovación de las líneas de productos se distinguen por sabores como: chocolate con trozos de café; con canela; con chile y otras mezclas como arándanos recubiertos de chocolate; además de que siguiendo las tendencias mundiales, procuran empaques novedosos. El chocolate para mesa usualmente se consume de manera líquida, mezclado con leche o con agua. Este es de gran arraigo familiar (figura $3)$.

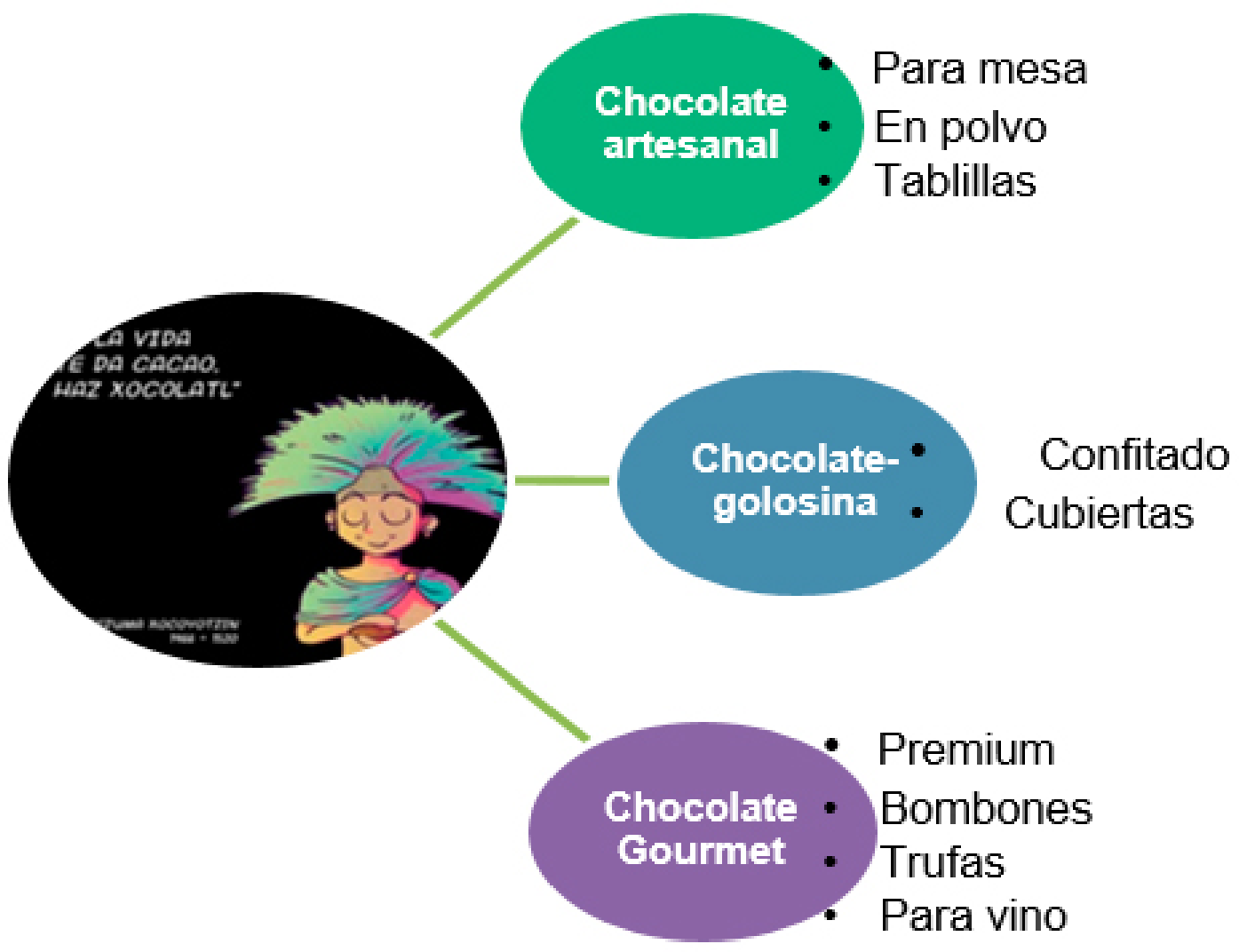

Figura 3. Principales tipos de chocolate fabricados en México. Fuente: Camacho (2017). 
La mayoría de las organizaciones analizadas elaboran productos con figuras alusivas a la cultura del lugar donde se encuentran ubicadas tales como piezas prehispánicas, conejos, osos, caracoles, flores, monedas y dominó, por mencionar las más conocidas.

\section{Precio}

Los precios de los diferentes tipos de chocolate fabricados por empresas mexicanas son fijados de manera competitiva, pero en algunos contextos son superiores al de la competencia internacional; particularmente en el chocolate para mesa, ya que su contenido de cacao es más alto. En el caso de los chocolates gourmet todas las empresas fijan precios similares, lo que obedece a que sus productos en general son diferenciados y de alta calidad. Consecuentemente se asume que sus estrategias de precios están orientadas a las ventas, buscando el crecimiento de ingresos y volúmenes de unidades y su incremento en la participación de mercados (figura 4).

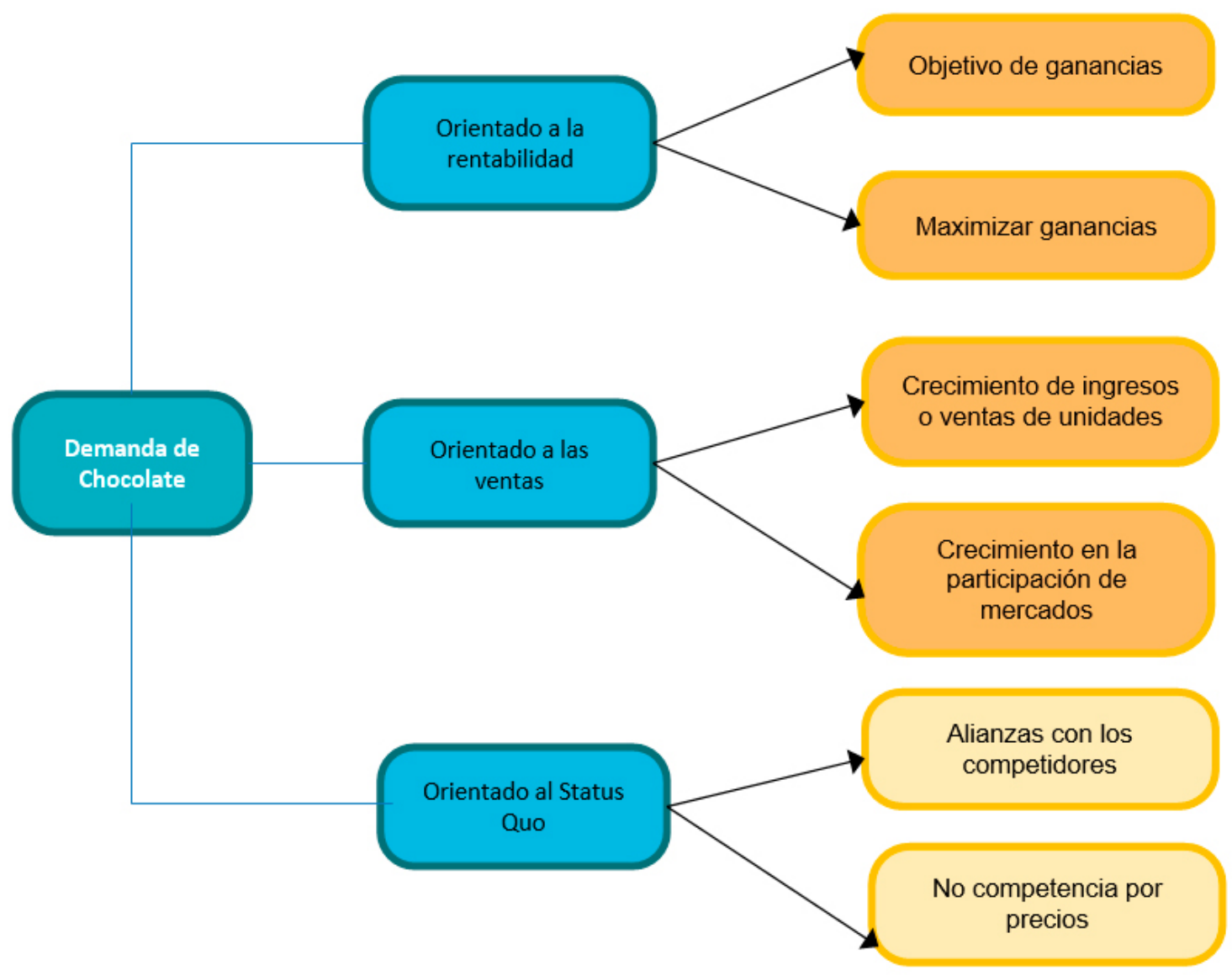

Figura 4. Posibles objetivos de precio para las pymes del Chocolate. Fuente: Perreault y McCarthy (2002). 


\section{Promoción de ventas}

En los chocolates la promoción es más efectiva cuando se encuentra vinculada o se complementa con estrategias publicitarias, siempre y cuando se definan previamente los objetivos de comunicación que las pymes consideren de acuerdo a sus segmentos y mercados. En México se estima que el período comprendido entre octubre y febrero es el de más altas ventas. Por ello las presentaciones de los envases y empaques cambian o se llevan a cabo diseños de colección; los precios también se incrementan, especialmente en temporadas navideñas (Camacho, 2017). De acuerdo a los datos localizados se aprecia que la promoción de ventas de estas empresas consisten en degustaciones en el punto de venta, aunque de manera especial lo hacen en festivales gastronómicos y ferias generales donde exhiben y comercializan sus productos; por ejemplo, los festivales de chocolate de reciente creación en México que al día de hoy suman nueve y que tienen lugar en ciudades como: Villahermosa, Tabasco; Coyoacán,
Ciudad de México; Puebla, Puebla; San Antonio Castillo Velasco, Oaxaca; Monterrey, Nuevo León y San Luis Potosí, San Luis Potosí.

\section{Publicidad}

La tendencia publicitaria predominante en las pymes del cacao y el chocolate es de boca en boca. El uso de medios tradicionales es limitado, aunque algunas de las empresas utilizan revistas especializadas para hacer presencia con sus productos. Sin embargo la práctica detectada es hacia la utilización de medios digitales a través de páginas Web propias o de externos, blogs, Facebook, Twitter, Instagram, algunas empresas se promueven a través de TripAdvisor -el sitio de viajes más grande del mundo que contiene más de 600 millones de opiniones de viajeros-; lo cual es una oportunidad para exhibir las marcas de chocolates mexicanos, que suelen ser adquiridos como recuerdo (tabla 3 , figura 5 ).

Tabla 3. Publicidad digital de las pymes mexicanas del chocolate

\begin{tabular}{|c|c|c|c|c|c|c|c|}
\hline Empresa & $\begin{array}{l}\text { Página } \\
\text { Web }\end{array}$ & $\begin{array}{c}\text { Blog o web de } \\
\text { terceros }\end{array}$ & Facebook & Twitter & Instagram & TripAdvisor & Newsletter \\
\hline Costanzo & $x$ & & $x$ & $x$ & $x$ & & \\
\hline Dos Hermanos & x Bilingüe & & $x$ & $x$ & $x$ & & \\
\hline Milan & $x$ & & $x$ & $x$ & $x$ & & \\
\hline Escalona & $x$ & & $x$ & $x$ & & & \\
\hline Moctezuma & $x$ & & $x$ & $x$ & & & \\
\hline Cacep & $x$ & & $x$ & $x$ & $x$ & & \\
\hline Ibarra & x Bilingüe & & $x$ & $x$ & $x$ & & \\
\hline La Popular & $x$ & & De terceros & & & & \\
\hline Turín & x Bilingüe & & $x$ & $x$ & $x$ & & \\
\hline Picarda & $x$ & & $x$ & $x$ & $x$ & & \\
\hline Rey Amargo & x Bilingüe & & $x$ & $x$ & $x$ & & \\
\hline La Suiza & $x$ & & $x$ & $x$ & $x$ & & \\
\hline Mayordomo & $x$ & & $x$ & $x$ & $x$ & & \\
\hline Wolter & $x$ & & $x$ & $x$ & $x$ & & $x$ \\
\hline Clatier & $x$ & & $x$ & $x$ & $x$ & & \\
\hline Continental & x Bilingüe & & $x$ & $x$ & $x$ & & \\
\hline Bets & $x$ & & & $x$ & $x$ & & \\
\hline kakaw & $x$ & $x$ & $x$ & $x$ & $x$ & $x$ & $x$ \\
\hline La Locomotora & & $x$ & & & & & \\
\hline Xocolatl & $x$ & $x$ & $x$ & $x$ & $x$ & $x$ & \\
\hline
\end{tabular}

Fuente: elaboración propia por parte de la autora. 


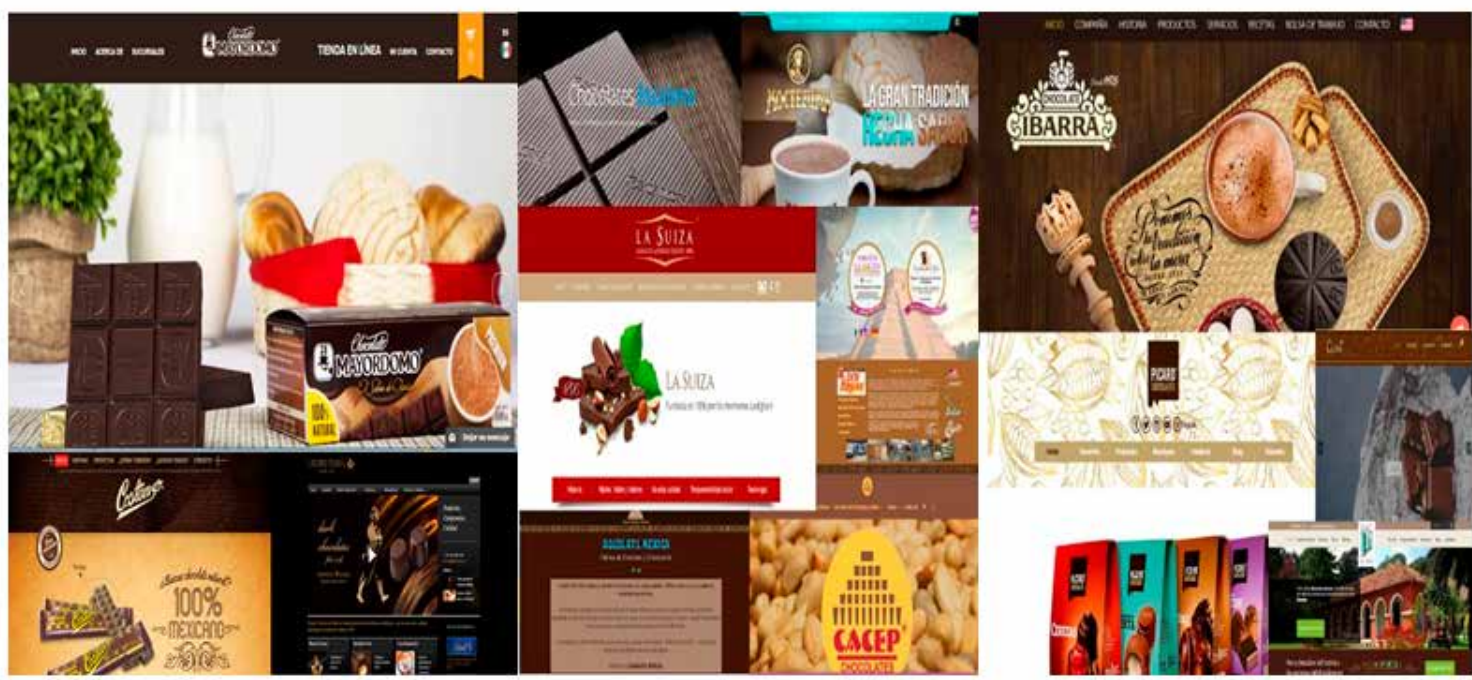

Figura 5. Homepage de las empresas mexicanas Mayordomo, Costanzo, Rey Amargo, Escalona, Moctezuma, La Suiza, Kakaw, Xocolatl Mexica, Cacep, Picard, Clatier, Wolter e Ibarra. Fuente: páginas Web de las empresas estudiadas.

Las páginas Web propias inciden en elementos tradicionales de la mexicanidad asociados con el chocolate artesanal tales como leche, pan, molinillo; y en el caso de Tabasco también se distinguen imágenes de las haciendas de cacao. Varias de las empresas impulsan la línea de productos gourmet cuya imagen se proyecta en sus mensajes publicitarios.

Otra forma de publicitarse es a través de reportajes o entrevistas concedidas a medios tradicionales como la televisión, radio y prensa. Revistas prestigiadas han sido grandes aliadas de las pymes del cacao y el chocolate como son México Desconocido, Expansión y National Geographic. Periódicos de diaria circulación como El Universal, El Periódico de México, Forbes y El Economista, algunos alentando el consumo de productos mexicanos y otros mostrando las ventajas de la ingesta de este producto.

Las prácticas del marketing en las pymes difieren de las aplicadas por la gran empresa, ya que sus iniciativas son limitadas en el plano operativo y planeación estratégica comercial. Sin embargo en el marketing del chocolate algunas acciones son convergentes. Un comparativo de publicidad y promoción de ventas en empresas grandes y pymes del chocolate en México evidencia las estrategias en función del tamaño del corporativo y el alcance de mercados que posee (tabla 4). 
Tabla 4. Comparativo de publicidad en promoción de ventas en grandes corporativos y pymes del chocolate en México

\begin{tabular}{|c|c|c|}
\hline & Publicidad & Promoción de ventas \\
\hline \multirow{7}{*}{$\begin{array}{l}\text { EMPRESA } \\
\text { GRANDE }\end{array}$} & $\begin{array}{l}\text { Campañas en redes sociales: Twitter, Facebook, Canal } \\
\text { YouTube Retail TouchPoints. }\end{array}$ & $\begin{array}{l}\text { Exposiciones en superficies comerciales para } \\
\text { la venta de productos }\end{array}$ \\
\hline & Spots televisivos y de radio. & \\
\hline & $\begin{array}{l}\text { Espectaculares, anuncios en parabuses, materiales } \\
\text { impresos, electrónicos. }\end{array}$ & Sorteos monetarios o de productos. \\
\hline & Publirreportajes, patrocinios, comunicados de prensa. & Cupones de descuento. \\
\hline & Campañas de relaciones públicas. & Tweets con premios. \\
\hline & Uso de personalidades artísticas o deportivas. & $\begin{array}{l}\text { Promoción con empaque para ediciones } \\
\text { especiales. }\end{array}$ \\
\hline & Campañas publicitarias de temporada. & $\begin{array}{l}\text { Estímulo al consumidor con comunicaciones } \\
\text { interactivas }\end{array}$ \\
\hline \multirow{7}{*}{ Empresa pyme } & $\begin{array}{l}\text { Anuncios en redes sociales: Facebook, Twitter, } \\
\text { Instagram. }\end{array}$ & Festivales del chocolate. \\
\hline & Páginas Web propias. & Rutas gastronómicas del chocolate \\
\hline & Páginas Web de terceros. & Catas y degustaciones esporádicas \\
\hline & Publireportajes. & \\
\hline & Volantes. & \\
\hline & Anuncios esporádicos en revistas & \\
\hline & Newsletter. & \\
\hline
\end{tabular}

Fuente: Camacho (2015).

Ventas

De acuerdo al tamaño de la empresa las ventas de los productos tienen básicamente ventas directas y ventas en línea. Sus procesos varían de una empresa a otra, aunque en general comercializan con pago en efectivo y donde sus tres segmentos clave son: distribuidores, residentes y turistas (figura 6).

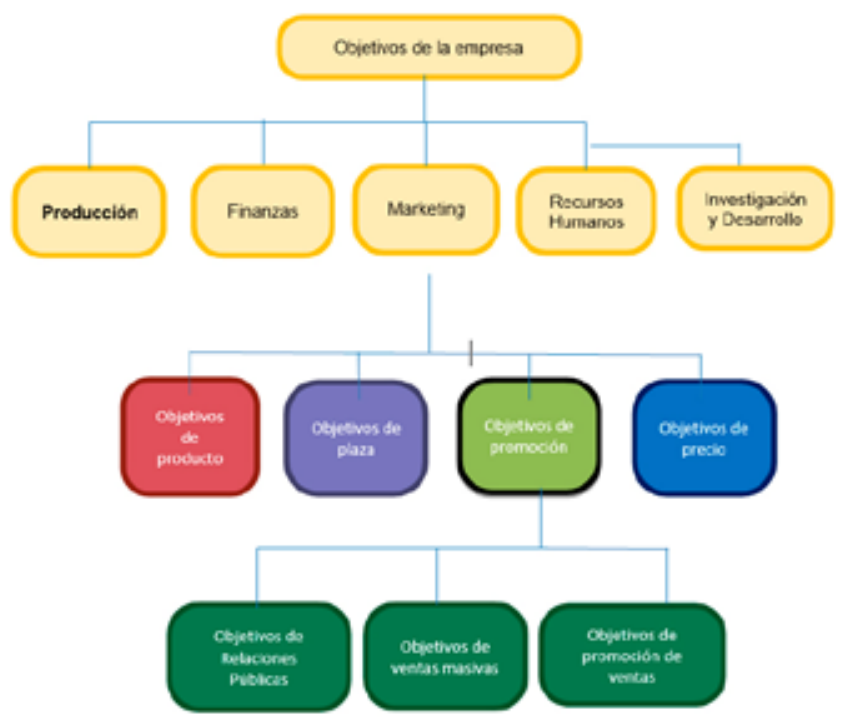

Figura 6. Jerarquía de objetivos por área funcional. Fuente: Perreault y McCarthy (2002, p. 97). 


\section{Relaciones públicas}

Los gerentes-propietarios tienden a establecer relaciones y alianzas con diversos públicos, con emprendedores de acciones que favorezcan sus negocios como es el caso del emprendimiento de festivales del chocolate. Algunas de las pymes ofrecen recorridos por sus fábricas, las cuales cuentan con tiendas donde distribuyen sus propios chocolates. Otras, como las ubicadas en Tabasco y Chiapas, ofrecen recorridos por las plantaciones de cacao.

En este sentido se identificó que tres pymes han impulsado la creación de museos del chocolate en los Estados de Tabasco, Chiapas y Yucatán (tabla 5).

Tabla 5. Museos del chocolate impulsado por pymes mexicanas

\begin{tabular}{llll}
\hline \multicolumn{1}{c}{ Empresa } & \multicolumn{1}{c}{ Ubicación } & \multicolumn{1}{c}{ Museo } & Fecha de apertura \\
\hline Chocolates Wolter & Comalcalco, Tabasco & $\begin{array}{l}\text { Museo vivo del cacao y el chocolate “Otto } \\
\text { Wolter Hayer }\end{array}$ & Noviembre de 2003 \\
\hline Kakaw chocolates & $\begin{array}{l}\text { San Cristóbal las Casas, } \\
\text { Chiapas }\end{array}$ & $\begin{array}{l}\text { Kakaw Museo del cacao y el chocolate y } \\
\text { chocolatería cultural Kakaw }\end{array}$ & Diciembre de 2010 \\
\hline Ki'Xocolatl & Tikul, Yucatán & Choco Story México-Ecomuseo del Cacao & Julio de 2014 \\
\hline
\end{tabular}

Fuente: elaboración propia por parte de la autora.

\section{Plaza}

Esta P's del marketing se encuentra muy vinculada a la producción de cacao a través de la geografía mexicana. De acuerdo con la Secretaría de Agricultura, Ganadería, Desarrollo Rural, Pesca y Alimentación -SAGARPA- los Estados de Chiapas, Tabasco, Oaxaca, Puebla y Veracruz constituyen la región estratégica. Esta zona está catalogada como una región prioritaria para el desarrollo de negocios del chocolate y donde estiman conveniente aplicar la estrategia de 'maximizar', que consiste en acciones clave como: "impulsar la adquisición de infraestructura y equipo para plantas productoras de chocolates artesanales y subproductos e implementar una campaña de promoción al consumo nacional y derivados del cacao" (SAGARPA, 2017, p. 10).

Tabasco y Chiapas son las entidades que más cacao producen en México, pero Tabasco es el líder con cerca del $70 \%$ del total de la producción (SAGARPA, 2017). Además, en 2016, obtuvieron la Declaración de Protección de Denominación de Origen para el Cacao Grijalva. Este reconocimiento añade valor a los productos elaborados con esta materia prima y facilita el acceso del cultivo tabasqueño y sus productos a mercados nacionales e internacionales, mejorando su rendimiento económico y elevando la competitividad local al ofrecer productos de calidad que sean reconocidos por su origen. Una de las características que otorga la identidad y la calidad del Cacao Grijalva es que se produce gracias al actuar conjunto de la naturaleza y el hombre en lo que se denominan selvas domesticadas (El Economista, 2016).

\footnotetext{
El Cacao Grijalva conserva un lugar privilegiado en la cultura de México pero, sobre todo, en Tabasco, lo que ha permitido que la conjugación de factores edafoclimáticos y la intervención del hombre en el manejo del cultivo; así como su proceso posterior a la cosecha, diferencian al grano del producido en otras zonas. (El Economista, 2016)
}

Durante la investigación se identificó la ubicación geográfica de las principales 21 pymes mexicanas del cacao y el chocolate, las cuales se localizan en: Michoacán; Jalisco; Aguascalientes; Nuevo León; San Luis Potosí; Veracruz; Chiapas; Tabasco; Yucatán; Oaxaca y Ciudad de México (tabla 6, figura 7). 
Tabla 6. Priancipales corporativos mexicanos del chocolate

\begin{tabular}{|c|c|c|c|}
\hline Empresa & Año de creación & Ubicación geográfica & Principales productos \\
\hline La Locomotora Chocolate & 1860 & Xalapa, Veracruz & Chocolate para mesa \\
\hline Chocolate Moctezuma & 1925 & Uruapan, Michoacán & $\begin{array}{l}\text { Chocolate para mesa, } \\
\text { chocolate en polvo, pasta de } \\
\text { cacao }\end{array}$ \\
\hline $\begin{array}{l}\text { Chocolatera de Jalisco, S.A. } \\
\text { de C.V (Chocolate Ibarra) }\end{array}$ & 1925 & Guadalajara, Jalisco & $\begin{array}{l}\text { Chocolate para mesa, } \\
\text { chocolate en polvo, } \\
\text { chocolate Premium }\end{array}$ \\
\hline Chocolates Turín & 1928 & Ciudad de México & Productos de chocolate \\
\hline $\begin{array}{l}\text { Fábrica de Chocolates la } \\
\text { Popular, S.A. de C.V. }\end{array}$ & 1932 & Monterrey, Nuevo León & $\begin{array}{l}\text { Productos de chocolate y } \\
\text { cacao, tablillas de chocolate }\end{array}$ \\
\hline Chocolate rey amargo & 1939 & Ciudad Guzmán, Jalisco & Chocolate para mesa \\
\hline La Suiza & 1943 & Ciudad de México & $\begin{array}{l}\text { Tablillas de chocolate, } \\
\text { chocolate confitado, } \\
\text { chocolate relleno, chocolate } \\
\text { para vino }\end{array}$ \\
\hline Chocolates Escalona & 1945 & Ciudad de México & Bombones y chocolates \\
\hline Chocolate Milán & 1946 & Ciudad de México & $\begin{array}{l}\text { Chocolate confitado, } \\
\text { bombones, trufas, chocolate } \\
\text { Premium }\end{array}$ \\
\hline Chocolate Dos Hermanos & 1948 & Guadalajara, Jalisco & $\begin{array}{l}\text { Chocolate para mesa, } \\
\text { chocolate en polvo }\end{array}$ \\
\hline Chocolate Mayordomo & 1956 & Tlacolula, Oaxaca & Chocolate para mesa \\
\hline Chocolate Imperial & 1956 & Mérida Yucatán & Chocolate para mesa \\
\hline Chocolates Wolter & 1958 & Comalcalco, Tabasco & $\begin{array}{l}\text { Chocolate para mesa, } \\
\text { chocolate en polvo, } \\
\text { bombonería, chocolate } \\
\text { Premium }\end{array}$ \\
\hline $\begin{array}{l}\text { Fábrica de chocolates y } \\
\text { dulces Costanzo, S.A. de } \\
\text { C.V. }\end{array}$ & 1958 & San Luis Potosí, SLP & $\begin{array}{l}\text { Chocolates, confituras de } \\
\text { chocolate }\end{array}$ \\
\hline ábrica de Chocolates Kakaw & 1958 & San Cristóbal las Casas, Chiapas & $\begin{array}{l}\text { Chocolate para mesa, } \\
\text { chocolate golosina, tabletas } \\
\text { de chocolate, bombones, } \\
\text { pralinés, trufas }\end{array}$ \\
\hline Chocolates El Chontal & 1958 & Comalcalco, Tabasco & $\begin{array}{l}\text { Chocolates para mesa, } \\
\text { tabletas de chocolate }\end{array}$ \\
\hline Cacep Chocolates & 1963 & Comalcalco, Tabasco & $\begin{array}{l}\text { Chocolate-golosina, } \\
\text { confiterías, chocolate } \\
\text { para mesa, coberturas de } \\
\text { chocolate, materia prima, } \\
\text { bebidas en polvo, figura } \\
\text { artesanales de chocolate }\end{array}$ \\
\hline $\begin{array}{l}\text { Continental Best, S.A. de } \\
\text { C.V. }\end{array}$ & 1964 & Ciudad de México & $\begin{array}{l}\text { Chocolates Premium, } \\
\text { bombones, trufas }\end{array}$ \\
\hline Picard Chocolates & 1964 & Ciudad de México & Chocolates Premium, trufas \\
\hline Xocolatl Mexica & 1989 & Aguascalientes & Chocolate para mesa \\
\hline Chocolates Clatier & 2010 & Mérida, Yucatán & Chocolates Premium, café \\
\hline
\end{tabular}

Fuente: Camacho (2017). 


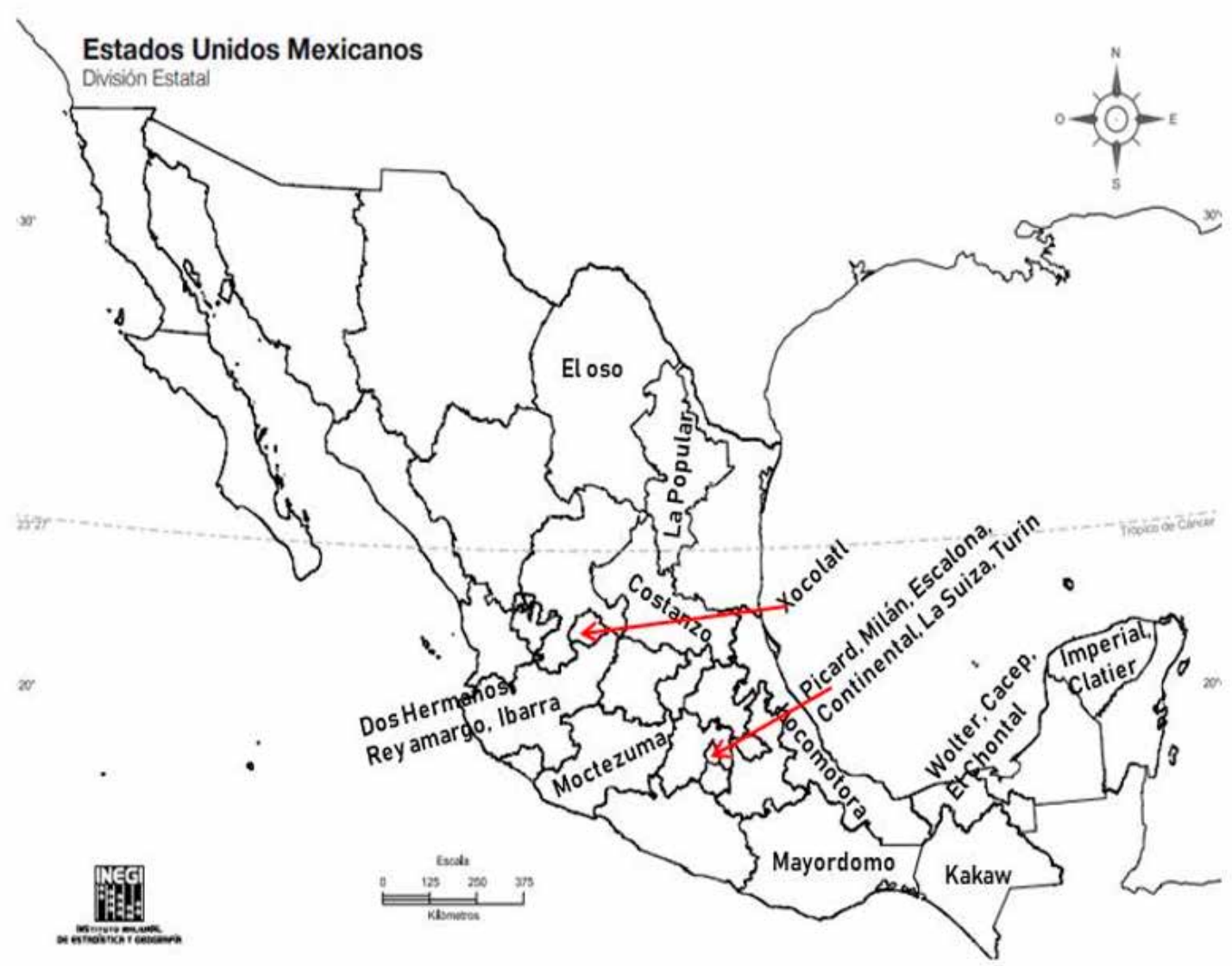

Figura 7. Ubicación de fábricas pymes de chocolate en México. Fuente: elaboración por parte de la autora a partir de INEGI (s.f.).

La distribución de la línea de productos de las empresas del chocolate es: puesto en fábrica; cadenas de autoservicio; tiendas de artesanías; mercados públicos; tiendas boutiques y tiendas virtuales; estas dos últimas corresponden más a las medianas organizaciones. Cinco de las empresas identificadas participan en mercados internacionales, exportan sus productos principales a Estados Unidos y Europa. Todas las pymes tienen puntos de venta propios. Los productos más comercializados son chocolate para mesa, en polvo, confitado, Premium, bombones y trufas.

Con esta investigación se evidencia la relevancia que tiene el proceso de producción en las fábricas y comercializadoras de cacao y chocolate. En consecuencia, los hallazgos sobre las principales acciones de marketing de las chocolateras mexicanas se concentran en tres P's: producto, promoción de ventas y publicidad y plaza (figura 8). 
- Chocolate para mesa

- Chocolate en polvo

- Tabletas (barras)

- Chocolate premium

- Mezclas de chocolate en

polvo con otros

ingredientes
Promoción de

Ventas y

Publicidad

- Redes sociales

-Página web propia

-Página web y blogs de

externos

- Newsletter

- Ferias y exposiciones

Línea de

productos
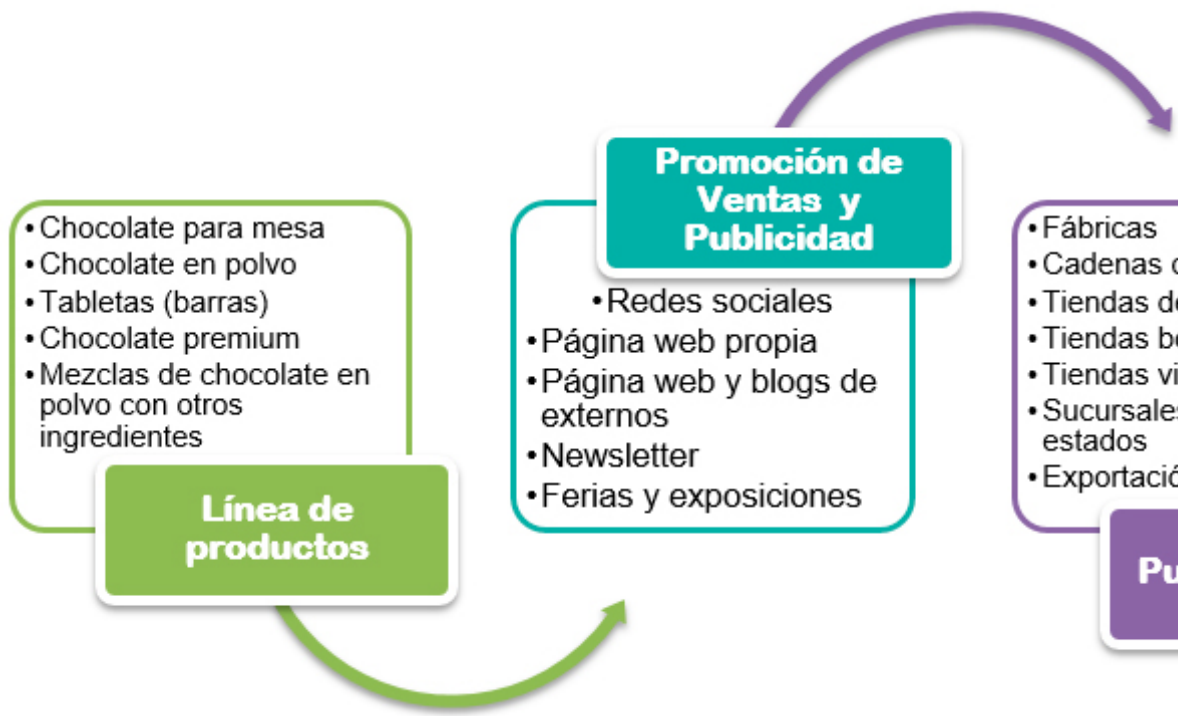

- Fábricas

- Cadenas de autoservicio

- Tiendas de artesanías

- Tiendas boutiques

- Tiendas virtuales

- Sucursales en otros estados

- Exportación

\section{Puntos de venta}

Figura 8. Estrategias de marketing en pymes del chocolate en México. Fuente: elaboración propia por parte de la autora.

\section{Conclusiones}

Con esta investigación fue posible analizar las principales estrategias de marketing de las pymes mexicanas que fabrican y comercializan chocolate; las cuales son de origen y capital mexicano. Se estudiaron un total de 21 organizaciones que se ubican en once entidades del país; de manera preponderante en las zonas del sureste, occidente y centro del país. El trabajo se desarrolló desde la perspectiva de las 4 P's del marketing (McCarthy, 1960; Perreault and McCarthy, 2002).

Las tendencias en estas empresas denotan prácticas del marketing en una dimensión básica con énfasis en el producto, promoción de ventas y plaza. Se observó una marcada mentalidad de producción; lo cual es una garantía de calidad del producto; empero, al momento del estudio no existía una clara visión del marketing que les ayudara a complementar y potencializar su proceso comercial (Reinares y Blanco, 2015). Generalmente este tipo de organizaciones no parecen identificar información comercial relevante dado que tienen escasa o nula utilización de nuevas tecnologías y no consolidan el diseño y calidad de sus productos en todas las etapas de la cadena de valor, por lo que sus marcas no alcanzan un nivel de desarrollo competitivo.
Siguiendo el modelo de las 4 P's del marketing fue posible conocer que en las 21 pymes mexicanas analizadas, el producto cuenta con tres principales líneas: chocolate para mesa, chocolate-golosina y chocolate gourmet. Otra característica de las pymes chocolateras es su participación en segmentos turísticos; en donde la mayoría de estas ofrecen una línea de servicios complementarios tales como museos del chocolate y chocolaterías. Tabasco y Chiapas también disponen de recorridos por las plantaciones de cacao que concluyen con degustaciones de chocolate.

El precio en general es superior al de los productos masivos con sabor a chocolate, justo por la materia prima que utilizan, los cuales son de mayor contenido de cacao y elaborados artesanalmente. Se identificaron estrategias de precios principalmente orientadas a las ventas, con miras al crecimiento de ingresos y volúmenes de unidades y al logro del incremento en la participación de mercados locales, nacionales e internacionales.

Con respecto a la promoción se aprecia la participación de las empresas en ferias gastronómicas especializadas donde dan a conocer sus productos tal como ocurre con los festivales del chocolate que tienen lugar en 12 ciudades; en donde, además de las pymes locales, participan organizaciones de otros países que tienen alta representatividad en 
el mercado del chocolate como Ecuador, Colombia y Venezuela, por citar solo algunos. En estos festivales también se dan cita chocolatiers y expertos nacionales e internacionales. La estrategia publicitaria dominante es de boca en boca. Si bien la utilización de medios tradicionales es limitada, las empresas pautan con revistas especializadas para hacer presencia con sus productos. La mayoría de las pymes del chocolate cuentan con páginas Web, en la generalidad de los casos son propias y en otras son propiedad de terceros (por ejemplo, organizaciones del sector como ASCHOCO), blogs de particulares, Facebook, Twitter, Instagram. De igual manera se detectó la publicidad de estas empresas en páginas como TripAdvisor, así como sitios relacionados con el turismo.

En cuanto a la plaza se localizaron geográficamente a 21 fábricas y comercializadoras de chocolate en el centro, occidente y sureste de México. El promedio de antigüedad de estas organizaciones es de 60 años, lo que evidencia la experiencia y conocimientos sobre este tipo de negocios. Se aprecia un cuidado intensivo en toda la cadena de suministro. En tanto a la distribución se identificaron diversos puntos de venta: puesto en fábrica; tiendas propias de los fabricantes; tiendas de autoservicio; tiendas de artesanías; tiendas boutique y tiendas en línea. Algunas de las medianas empresas exportan sus productos a diversos países, especialmente a Europa y los Estados Unidos. La forma de distribución está estrechamente ligada a la ubicación geográfica, particularmente por sus volúmenes de producción asociados a condiciones naturales del lugar.

Con este artículo se puede confirmar que tal como ocurre con el sector agroindustrial, las tendencias de las pymes mexicanas (productoras y comercializadoras de chocolate) muestran la prioridad por los procesos de siembra-cosecha y la producción; en tanto que el marketing aún representa un área de oportunidad para emprender acciones estratégicas que les permitan colocar y posicionar sus marcas entre consumidores intermedios y clientes finales. Los gerentes-propietarios son conocedores del negocio y de los mercados nacionales e internacionales, lo que representa una gran ventaja. Sin embargo aún no emprenden estrategias innovadoras contundentes para atender de forma competitiva y diferenciada a sus mercados y segmentos.

\section{Referencias}

Barrow, C. (1996). La esencia de la administración de pequeñas empresas. Ciudad de México, México: Prentice-Hall Hispanoamericana.

Camacho, M. (2014). Chocolate del edén para el mundo. Tabasco, México: Consejo Coordinador Empresarial, Universidad Juárez Autónoma de Tabasco.

Camacho, M. (2015). Marketing del chocolate en México. VinculaTégica EFAN, 1 (1).

Camacho, M. (2017). Chocolate. Historia, arte, cultura y marketing. Tendencias mundiales. Ciudad de México, México: Editorial Laberinto.

Camacho, M. (2018). Competencias directivas del empresario agroindustrial. Pensamiento y Gestión, 44, 13-43.

Camacho, M. y Andrade, J. (2015). Mercadotecnia en perspectiva. Reflexiones y casos. Ciudad de México, México: Editorial Trillas.

El Economista. (12 de mayo de 2016). El chocolate es mexicano, pero en México se consume poco. Recuperado de https://www.eleconomista.com.mx/empresas/El-chocolate-es-mexicano-pero-en-Mexico-se-consume-poco-20150912-0007.html.

Equipo Vértice. (2009). La gestión del marketing, producción y calidad en las PYMES. Málaga, España: Publicaciones Vértice.

FAO. (2013). Agroindustrias para el desarrollo. Roma Italia: FAO.

Gobmx. (8 de mayo de 2017). Agroindustria en México. Recuperado de https://www.gob.mx/firco/articulos/agroindustria-en-mexico?idiom=es.

INEGI. (Sin fecha). Información para niños y no tan niños. Recuperado de http://cuentame.inegi.org. $\mathrm{mx} / \mathrm{mapas} /$ nacional.aspx?tema=M.

INEGI. (2009). Micro, pequeña, mediana y gran empresa. Censos Económicos 2009. Ciudad de México, México: INEGI.

Kotler, P. y Armstrong, G. (2001). Marketing. Ciudad de México, México: Prentice Hall.

Marcos, J. (2015). ¿Qué es eso del marketing? Guía práctica para PYMES. Buenos Aires, Argentina: Errepar S.A. 
Martínez, M. (2006). Ciencia y arte en la metodología cualitativa. Ciudad de México, México: Editorial Trillas.

McCarthy, E.J. (1960). Basic marketing: A management approach. New York, USA: McGraw-Hill Education.

Perreault, W.D. and McCarthy, E.J. (2002). Basic Marketing a Global-Managerial Approach. New York, USA: McGraw-Hill Higher Education.

PyME-JICA. (2005). Formación de consultores PYME 2005. Módulo VII Mercadotecnia. Ciudad de México, México: CANACINTRA.

Quadratín. (26 de febrero de 2018). Necesario, fortalecer la industria del chocolate en México. Recuperado de https://www.quadratin.com.mx/economia/ necesario-fortalecer-la-industria-del-chocolate-en-mexico/.

Reinares, E. y Blanco, A. (2015). La gestión del marketing en las PYMES. Madrid, España: Cátedra Madrid Excelente.

Resnick, S.M. et al. (2016). Marketing in SMEs: A "4Ps" self branding model. International Journal of Entrepreneurial Behavior \& Research, 22 (1), 155-174.

SAGARPA. (2017). Planeación Agrícola Nacional 20172030. Ciudad de México, México: SAGARPA.

Secretaría de Economía. (Sin fecha). Medianas empresas. Recuperado de http://www.2006-2012. economia.gob.mx/mexico-emprende/empresas/ mediana-empresa.

Secretaría de Economía. (2016). Diagnóstico 2016 del Fondo Nacional Emprendedor. Ciudad de México, México: Secretaría de Economía, Instituto Nacional del Emprendedor. 